\title{
The prevalence, clinical features, predictive factors and investigations to screen for cancer in patients with inflammatory myositis
}

\author{
A case series from two tertiary care centers in Riyadh, Saudi Arabia
}

Ghassan Aljohani, MBBS, Eman A. Bin Awad, MBBS, Khalid Alshahrani, MBBS, Mubark M. Alsaqar, MBBS,

Bashayer Albogami, MBBS, Saleh H. Almotywee, MBBS, Hisham Almaimouni, MBBS, Abdullah S. Dirar, MBBS,

Abdulrahman Alrashid, MBBS, Rajkumar Rajendram, MBBS, Emad Masuadi, MBBS, Mohammed A. Omair, MBBS.

\begin{abstract}
الأهداف : وصف وبائيات حدوث السرطان في المرضى الذين يعانون من التهاب

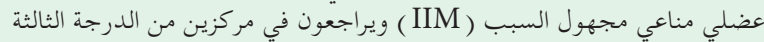
في الرياض، ألمملكة العربية السعودية.

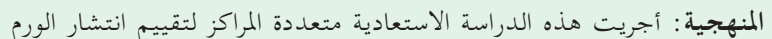

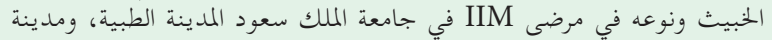

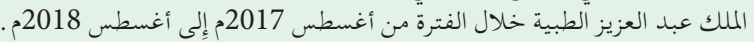

النتائج: اشتملت الدراسة على 60 مريضاً. من بينهم، 4 مصابون بأورام بـوام

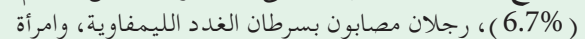

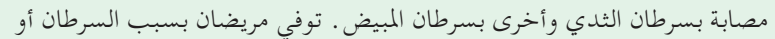

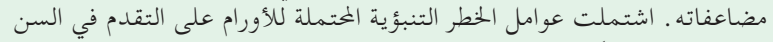

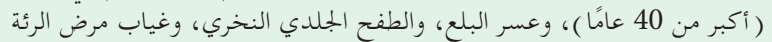

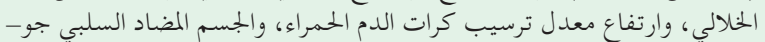

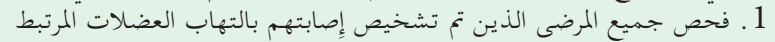

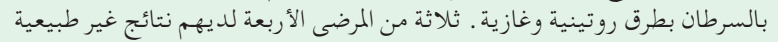

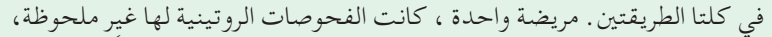

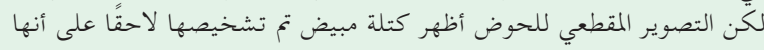

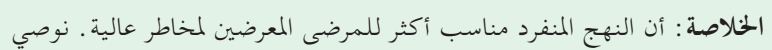

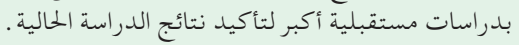

Objectives: To describes the epidemiology of cancer in patients with idiopathic inflammatory myopathies (IIM) treated at 2 tertiary centers in Riyadh, Saudi Arabia.

Methods: This was a retrospective multi-center study evaluating the prevalence and the type of malignancy in an IIM population in King Saud University Medical City and King Abdulaziz Medical City between August 2017 to August 2018.

Results: In total, 60 patients were included. Four had neoplasms (6.7\%), 2 men had lymphoma, a woman had breast cancer and a second, ovarian cancer. Two patients died due to cancer or its complications. Older age (age $>40$ years), dysphagia, necrotic rash, absence of interstitial lung disease, high erythrocyte sedimentation rate and a negative anti Jo-1 antibody were potentially predictive risk factors for neoplasm. All patients diagnosed with cancer-associated myositis were investigated with routine and invasive modalities. Three of the 4 patients had abnormal findings in both modalities. One patient, the routine investigations were unremarkable, but a computed tomography of the pelvis revealed an ovarian mass that was subsequently diagnosed as malignant.

Conclusion: An individualized approach might be more appropriate for high risk patients. Larger prospective studies are required to confirm the findings of the current study.

Keywords: dermatomyositis, polymyositis, malignancy, cancer, screening, inflammatory myopathies

Saudi Med J 2021; Vol. 42 (1): 100-104

doi: 10.15537/smj.2021.1.25590

From the Department of Medicine (Aljohani, Bin Awad, Alsaqar, Albogami, Almotywee, Almaimouni, Dirar, Alrashid, Rajendram, Masuadi), King Abdulaziz Medical City, Ministry of National Guard - Health Affairs; from the College of Medicine (Aljohani, Bin Awad, Alsaqar, Albogami, Almotywee, Almaimouni, Dirar, Alrashid, Rajendram, Masuadi), King Saud Bin Abdulaziz University for Health Sciences; from the King Abdullah International Medical Research Center (Aljohani, Bin Awad, Alsaqar, Albogami, Almotywee, Almaimouni, Dirar, Alrashid, Rajendram, Masuadi); and from the Rheumatology Unit (Alshahrani, Omair), Department of Medicine, King Saud University, Riyadh, Kingdom of Saudi Arabia.

Received 1st August 2020. Accepted 23rd November 2020.

Address correspondence and reprint request to: Dr. Mohammed A. Omair, Rheumatology Division, Department of Medicine, College of Medicine, King Saud University, Riyadh, Kingdom of Saudi Arabia. E-mail:momair@ksu.edu.sa

ORCID ID: http://orcid.org/0000-0002-9373-5473 
diopathic inflammatory myopathies (IIM) are 1 subdivided in 3 categories, including adult dermatomyositis (DM), polymyositis (PM), and inclusion body myositis (IBM). The old standard for the diagnosis of IIM is the Bohan and Peter diagnostic criteria using clinical, laboratory, and pathological features. ${ }^{1,2}$ It is known that inflammatory myopathy (IM) may be a paraneoplastic phenomenon and its association with malignancy is a poor prognostic factor and correlates with mortality. ${ }^{3}$ The prevalence of neoplasms in patients with IM varies in the literature. There are some studies from Asia, Australia, China, and Malaysia; however, the prevalence in the Middle East remains uncertain. In Saudi Arabia, only one case series of 22 patients reported malignancy identified in 5 women $(22.7 \%)$ and the mortality rate due to malignancy was $4.5 \% .{ }^{4}$ We investigated the prevalence and type of malignancy in IIM patients receiving treatment at 2 tertiary referral centers in Riyadh, Saudi Arabia. We also evaluated the clinical and laboratory findings which may predict the risk of cancer and assessed the investigations used to screen for malignancy in this cohort.

Methods. This was a retrospective multi-center study evaluating the prevalence and the type of malignancy in an IIM population in King Saud University Medical City and King Abdulaziz Medical City between August 2017 to August 2018. Ethical approval for this study was obtained from the Institutional Review Boards of King Abdullah International Medical Research Center and King Saud University Medical City. This retrospective chart review included 60 patients attending 2 tertiary care centers in Riyadh, Saudi Arabia. All were diagnosed with either DM or PM according to the American College of Rheumatology/European League Against Rheumatism classification criteria ${ }^{5}$ and had a clinical or histopathological diagnosis consistent with IM. Patients with juvenile myositis, IBM, drug induced myositis, overlap myositis and a diagnosis of malignancy more than 2 years prior to the diagnosis of myositis were also excluded.

The medical files of each patient were retrieved from the Records Departments of both hospitals. A prepared

Disclosure. This study was supported by the College of Medicine Research Centre and the Deanship of Scientific Research of King Saud University, Riyadh, Saudi Arabia. Authors have no conflict of interests, and the work was not supported or funded by any drug company. paper-based instrument was used to standardize data collection. The primary outcome was the development of malignancy within 2 years before or 5 years after the diagnosis. In addition to demographic information, data related to symptoms (proximal weakness, necrotic or atypical rash, dysphagia, arthralgia), signs (interstitial lung disease (ILD), Raynaud phenomenon, mechanic's hands, fever), laboratory results (urine analysis, anemia, cholestasis, C-reactive protein and erythrocyte sedimentation rate, serum creatine kinase, anti Jo-1 antibodies, antinuclear antibodies, complement C4) and pathology (muscle biopsy), and treatment was collected. Other myositis specific autoantibodies were not tested.

The investigations used to screen for malignancy included laboratory and radiological tests, including a chest $\mathrm{x}$-ray and computed tomography (CT) of the chest, abdominal and pelvis, thyroid ultrasound, upper gastrointestinal endoscopy, and colonoscopy in patients with a positive fecal occult blood test. In addition, a breast ultrasound, mammogram and CA125 were carried out for women and prostate-specific antigen (PSA) for men as well as a DEXA bone density scan. Interstitial lung disease was diagnosed by high-resolution CT of the chest. Dysphagia was defined by difficulty in swallowing water or food, barium swallow findings or the requirement for a nasogastric tube feeding after exclusion of all other causes. If the patient was found to have a malignancy, additional data was collected on the date of diagnosis of the malignancy regarding the diagnosis of IIM and the site of the malignancy. The data were compared with the historical data from Saudi Arabia reported by Al-Ballaa et al. ${ }^{4}$

Data were analyzed using the Statistical Package for Social Sciences version 20.0 (Armonk, NY: IBM Corp.). Categorical data were presented as frequency and percentage. Nominal data were presented as mean and standard deviation. A Fisher exact test was used to overcome the relatively small sample size. Statistical significance was accepted if the $p$-value $\leq 0.05$.

Results. A total of 80 patient files were reviewed of which 20 patients did not fulfill the inclusion criteria. Finally, 60 patients were included in this case series, 45 patients from the first institution and 15 from the second institution. Dermatomyositis was diagnosed in 31 cases with 29 cases of PM. The age ranged from 19 years to 85 years. Distribution included 16 (26.7\%) patients were less than 40 years, while 17 (28.3\%) were older than 60 years and the remaining $(45 \%)$ were between 40-59 years of age. Forty-six (76.7\%) were females. Of the 60 IIM cases, 2 DM cases had cancer, one breast cancer and the second lymphoma. In the PM 
group, there was one case of ovarian cancer and one case of lymphoma. In total, 4 cases $(6.7 \%, p=0.0536)$, which was not statistically different from the previous data from Saudi Arabia reporting malignancies in 5 of $22(22.7 \%)$ cases with IIM.

The most frequent presentation of IIM in this cohort included arthritis and polyarthropathy (60\%), fever $(35 \%)$ and rash (28\%). Dysphagia (20\%), interstitial lung disease (25\%), Raynaud phenomenon (3\%) and mechanical hands (2\%) were less frequent.

Regarding the clinical presentation of the 4 cases with malignancy, 2 had dysphagia, 3 presented with fever and one had an atypical rash. None of the patients with malignancy had ILD. These variables were not predictive of a risk of neoplasm in patients with IIM (Fishers exact test, $p>0.05$ ).

The diagnosis of IIM was confirmed pathologically in 65\%. Antinuclear antibodies were present in $90 \%$ and in the majority, the inflammatory markers were elevated (ESR, 61\%; CRP, 56\%). In 47\%, the CK activity was increased, $43.3 \%$ were anemic and $26.7 \%$ had cholestatic liver function tests. However, only 6
(10\%) had a positive anti Jo-1 and $8.3 \%$ had a low complement $c 4$. The urine analysis was abnormal in only $6.7 \%$.

Antinuclear antibody was positive in all 4 patients with neoplasia associated with IIM and the CK activity was high. Three cases were anemic, 50\% had cholestasis and hematuria with the anti Jo-1 positive in one case.

All patients with neoplasia had a pathological diagnosis of malignancy. Malignancy was diagnosed 0-5 years after the diagnosis of IIM. Two cases were diagnosed with malignancy at presenting with IIM and 2 cases died of malignancy.

The most frequent investigation used to screen for malignancy was chest radiography (98\%), DEXA scan $(48 \%)$, pan CT $(47 \%)$, and fecal occult blood (38\%). Tumor markers, mammogram and EGD were performed less often (28\%-36\%). A thyroid ultrasound $(12 \%)$ and colonoscopy (10\%) were infrequently requested (Figure 1). The malignancy cases in the current study have been evaluated with routine and invasive modalities. Three out of $4(75 \%)$ cases had abnormal findings in both modalities, but one patient

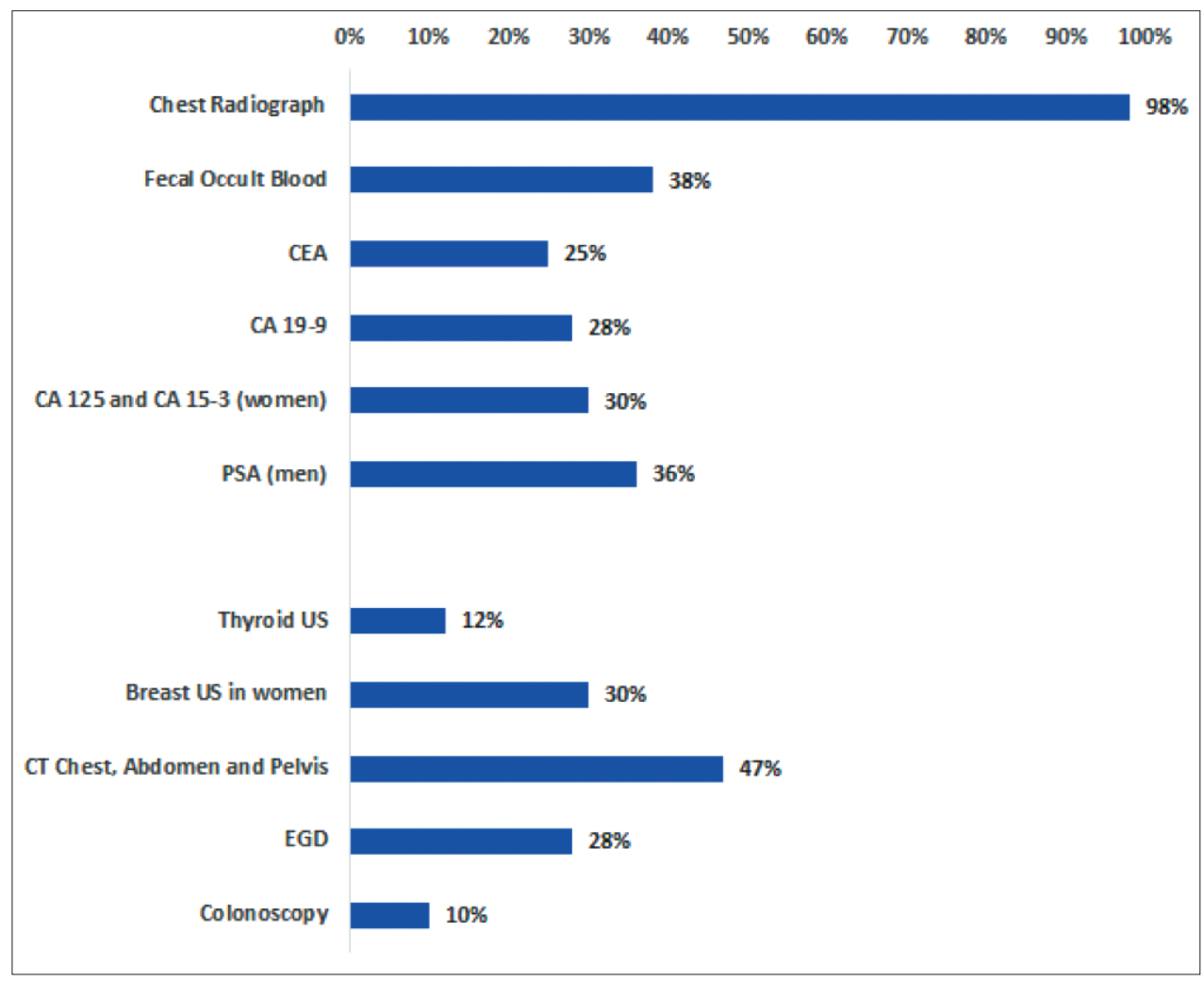

Figure 1 - Cancer diagnostic screening tests performed and their frequency. CEA: carcinoembryonic antigen, CA 19-9: cancer antigen 19-9, CA 125: cancer antigen 125, PSA: prostate-specific antigen, EGD: esophagogastroduodenoscopy 
had unremarkable routine investigations. However, a CT of the pelvis revealed an ovarian mass, diagnosed as ovarian cancer.

All patients received corticosteroids as first line therapy. However, the second line treatment varied. The majority of the cases received azathioprine (38\%), mycophenolate mofetil (30\%), and methotrexate (23\%). Immunoglobulins (3.3\%) and rituximab (5\%) were less frequently prescribed.

Discussion. Several studies investigated this phenomenon. However, the data vary greatly. For example, of 94 patients with IIM in Jordan, 4 (4.3\%) were diagnosed with malignancy. ${ }^{6}$ However, an Australian retrospective chart review reported malignancy in 21\% of 373 patients diagnosed with IIM between 1980 and 2009. ${ }^{7}$ Resources and advances in technology will significantly improve the screening of patients with malignancy. Though not statistically significant, it is noteworthy that a quarter of a century after Al-Ballaa et al. reported malignancies in $5(22.7 \%)$ of 22 patients with IIM in Saudi Arabia; malignancy was identified in only $6.7 \%$ in the current cohort. ${ }^{4}$ We believe that the previous study overestimated malignancy prevalence due to small sample size.

In the current case series, there was no difference in the risk of developing malignancy in terms of gender. In contrast to the previous Saudi study. ${ }^{4}$ Literature also reports contradictory gender findings. Several studies from Australia, ${ }^{7}$ Malaysia, ${ }^{8}$ and Japan, ${ }^{9}$ reported the male gender as a risk factor, though case series from South India, ${ }^{10}$ and Korea. ${ }^{11}$

Older age at presentation with IIM is considered a predictive risk factor for the association of cancer with IIM. In our cohort, all patients with cancer were over 40 years of age. This observation is consistent with data from Asian and Western populations. ${ }^{8-12}$

Lymphoma was the most frequent malignancy observed in our cohort, with both patients were males. Breast and ovarian cancer were diagnosed in the women. Other malignancies reported in patients with IIM include lung, prostate, colon, pancreas and other hematological malignancies. ${ }^{12-14}$

Similar to data from previous studies, ${ }^{15}$ the spatial relation between IIM and malignancy varies with two patients were diagnosed with cancer within 12 months of their diagnosis with IIM, one after 3 years and the fourth, 5 years after the initial presentation.

Fever, arthralgia and rash were the most frequent presenting symptoms in our cohort of IIM patients. Of the four patients with cancer, none had ILD and two had dysphagia. Dysphagia and the absence of ILD have been reported as predictive of cancer in patients with IIM. ${ }^{15}$ All four patients with cancer in our cohort had a high ESR and increased CK activity; ANA was positive in three and anti Jo- 1 antibodies were absent. The association of these findings with increased risk of malignancy are supported in literature. ${ }^{15}$

The investigations most frequently used for screening were chest x-ray, pan CT and faecal occult blood. Endoscopy, thyroid and breast imaging were performed in a small proportion $(<30 \%)$. All four patients who were diagnosed with cancer-associated myositis in our cohort, were investigated with routine and invasive tests. The results of the routine investigations were abnormal in three patients. However, in one case, despite the normal results of the routine investigations, a CT pelvis revealed an ovarian mass that was subsequently found to be ovarian cancer.

Our observations are consistent with literature ${ }^{16}$ emphasizing the importance of extensive screening for cancer at the diagnosis with IIM and during follow-up. However, there are no clear guidelines on which investigations should be performed to screen for malignancy in patients with IIM. The American Cancer Society (ACS) recommends screening for breast cancer in women of average risk from the age of 45 years. ${ }^{17}$ However, breast cancer has previously been reported in patients with IIM younger than 45 years. ${ }^{18}$ In Saudi Arabia, the mean age of breast cancer diagnosis is $55.93 \pm 15.96$ years which is one decade older than our patient. ${ }^{19}$

In terms of treatment received, all patients in our cohort received steroids, but the second line immunosuppression therapy varied. Azathioprine, mycophenolate mofetil and methotrexate were most frequently used. Mycophenolate mofetil was often used in patients with associated malignancies.

The mortality in our case series due to malignancy was high. Of the four patients with cancer, two patients died from cancer related complications. However, 29 patients without cancer died from infection or the progression of ILD, supported by literature reporting infection and ILD as the most frequent causes of mortality in patients with IIM..$^{20,21}$

Study limitation of our study include small sample size, unavailability of non-Jo-1 antibodies and lack of a control population.

In conclusion, the prevalence of cancer in our cohort was $6.7 \%$. Lymphoma was the most frequent neoplasm, followed by breast and ovarian cancer. The mean time of diagnosis with malignancy was 12 months after the onset of IIM. Older age, dysphagia, necrotic rash, absence of ILD, high ESR, and a negative anti Jo-1 are potential predictive risk factors for the development of neoplasia. The diagnosis of breast cancer in a 
47-year-old woman mandates that 'age appropriate' criteria for cancer screening should not be applied to patients with IIM. The development of an international consensus guideline for screening patients with IIM for cancer would be beneficial to this high-risk population.

This study was supported by the College of Medicine Research Centre and the Deanship of Scientific Research of King Saud University, Riyadh, Saudi Arabia.

Acknowledgement. This study was supported by the College of Medicine Research Centre and the Deanship of Scientific Research of King Saud University, Riyadh, Saudi Arabia.

\section{References}

1. Bohan A, Peter JB. Polymyositis and dermatomyositis (second of two parts). N Engl J Med 1975; 292: 403-407.

2. Bohan A, Peter JB. Polymyositis and dermatomyositis (first of two parts). N Engl J Med 1975; 292: 344-347.

3. Buchbinder R, Hill CL. Malignancy in patients with inflammatory myopathy. Curr Rheumatol Rep 2002; 4: 415-426.

4. Al-Ballaa ST, Al-Dalaan AN, El-Ramahi KM, Al-Janadi MA, Al-Shaikh A, Bahabri S. Pattern of adult onset of polymyositis and dermatomyositis and association with malignancy. Ann Saudi Med 1993; 13: 525-529.

5. Lundberg IE, Tjarnlund A, Bottai M, Werth VP, Pilkington C, Visser M, et al. 2017 European League Against Rheumatism/ American College of Rheumatology classification criteria for adult and juvenile idiopathic inflammatory myopathies and their major subgroups. Ann Rheum Dis 2017; 76: 1955-19564.

6. Mustafa KN, Al-Heresh AM, Khataybeh OY, Alawneh KM, Khader YS. Low prevalence of malignancy in patients with idiopathic inflammatory myopathies in Jordan. Clin Exp Rheumatol 2015; 33: 731-733.

7. Limaye V, Luke C, Tucker G, Hill C, Lester S, Blumbergs $\mathrm{P}$, et al. The incidence and associations of malignancy in a large cohort of patients with biopsy-determined idiopathic inflammatory myositis. Rheumatol Int 2013; 33: 965-971.

8. Tang MM, Thevarajah S. Paraneoplastic Dermatomyositis: A 12-year Retrospective Review in the Department of Dermatology Hospital Kuala Lumpur. Med J Malaysia 2010; 65: 138-142.

9. Azuma K, Yamada H, Ohkubo M, Yamasaki Y, Yamasaki $\mathrm{M}$, Mizushima $\mathrm{M}$, et al. Incidence and predictive factors for malignancies in 136 Japanese patients with dermatomyositis, polymyositis and clinically amyopathic dermatomyositis. Mod Rheumatol 2011; 21: 178-183.
10. Kannan MA, Sundaram C, Uppin M, Mridula R, Jabeen SA, Borgohain R. Incidence of malignancies in biopsy-proven inflammatory myopathy. Neurol India 2013; 61: 152-155.

11. Lee SW, Jung SY, Park MC, Park YB, Lee SK. Malignancies in Korean patients with inflammatory myopathy. Yonsei Med J 2006; 47: 519-523.

12. Andras C, Ponyi A, Constantin T, Csiki Z, Szekanecz E, Szodoray P, et al. Dermatomyositis and polymyositis associated with malignancy: a 21-year retrospective study. J Rheumatol 2008; 35: 438-444.

13. Chen YJ, Wu CY, Huang YL, Wang CB, Shen JL, Chang YT. Cancer risks of dermatomyositis and polymyositis: a nationwide cohort study in Taiwan. Arthritis Res Ther 2010; 12: R70.

14. Hill CL, Zhang Y, Sigurgeirsson B, Pukkala E, Mellemkjaer L, Airio A, et al. Frequency of specific cancer types in dermatomyositis and polymyositis: a population-based study. Lancet 2001; 357: 96-100.

15. Lu X, Yang H, Shu X, Chen F, Zhang Y, Zhang S, et al. Factors predicting malignancy in patients with polymyositis and dermatomyostis: a systematic review and meta-analysis. PLoS One 2014; 9: e94128.

16. Leatham H, Schadt C, Chisolm S, Fretwell D, Chung L, Callen JP, et al. Evidence supports blind screening for internal malignancy in dermatomyositis: Data from 2 large US dermatology cohorts. Medicine (Baltimore) 2018; 97: e9639.

17. Oeffinger KC, Fontham ET, Etzioni R, Herzig A, Michaelson JS, Shih YC, et al. Breast Cancer Screening for Women at Average Risk: 2015 Guideline Update From the American Cancer Society. JAMA 2015; 314: 1599-1614.

18. Hendren E, Vinik O, Faragalla H, Haq R. Breast cancer and dermatomyositis: a case study and literature review. Curr Oncol 2017; 24: e429-e433.

19. Asiri S, Asiri A, Ulahannan S, Alanazi M, Humran A, Hummadi A. Incidence Rates of Breast Cancer by Age and Tumor Characteristics Among Saudi Women: Recent Trends. Cureus 2020; 12: e6664.

20. Woo JH, Kim YJ, Kim JJ, Choi CB, Sung YK, Kim TH, et al. Mortality factors in idiopathic inflammatory myopathy: focusing on malignancy and interstitial lung disease. Mod Rheumatol 2013; 23: 503-508.

21. Xiao Y, Zuo X, You Y, Luo H, Duan L, Zhang W, et al. Investigation into the cause of mortality in 49 cases of idiopathic inflammatory myopathy: A single center study. Exp Ther Med 2016; 11: 885-889. 